\title{
Charting the neighbourhood
}

\author{
Rami Rekola ${ }^{1}$, Helmut Jerjen ${ }^{2}$ \\ and Chris Flynn ${ }^{1}$ \\ ${ }^{1}$ Tuorla Observatory, VISPA, University of Turku, Piikkiö, FI-21500, Finland \\ email: rareko@astro.utu.fi \\ ${ }^{2}$ Research School of Astronomy and Astrophysics, The Australian National University, \\ Mt Stromlo Observatory, Cotter Road, Weston ACT 2611, Australia
}

\begin{abstract}
We present Surface Brightness Fluctuation (SBF) distances of fifteen early-type dwarf galaxies and the S0 galaxy NGC 4150 in the Local Volume based on deep $B$ - and $R$-band CCD images obtained with the 2.56 metre Nordic Optical Telescope (NOT). Distances have been determined from Fourier analysed fields selected from optimal locations on the galaxies. Distances were found to range from 3 to $16 \mathrm{Mpc}$.

The SBF method has been demonstrated to efficiently measure relatively accurate distances from the ground with mid-aperture telescopes. The SBF method to measure distances to dwarf elliptical $(\mathrm{dE})$ galaxies has also been empirically calibrated using three dEs with known tip of the red giant branch (TRGB) distances.
\end{abstract}

Keywords. galaxies: clusters: individual (M81 group, M101 group, CVn I cloud, CVn II cloud, Leo I group, Coma I group), galaxies: dwarf, galaxies: stellar content, galaxies: structure, galaxies: distances and redshifts

\section{Introduction}

Recent imaging surveys of the Local Group (LG) neighbourhood have found and identified a large number of low surface brightness galaxy candidates, which potentially could be nearby dwarf galaxy systems (e.g. Côté, Freeman, Carignan \& Quinn (1997); Karachentseva \& Karachentsev (1998); Jerjen, Binggeli \& Freeman (2000)). Many are dEs located in galaxy groups as satellites of giant galaxies. Whether dEs are local or in the background has cosmological implications. For example Moore, Ghigna, Governato, et al. (1999) have numerically studied galactic and cluster halo substructures in a hierarchical universe, and found that simulations predict an abundance of Milky Way dwarf satellites currently unobserved. This conflict may be partly addressed by catalogueing and obtaining accurate distances of dwarf galaxy populations in the local universe. As a new and numerous target group in extragalactic studies dEs serve also to test the peculiar linearity and smoothness of the Hubble flow in the local volume. As such they may help to define the dark energy solution to the problem (Baryshev, Chernin \& Teerikorpi (2001)).

\section{Distances}

We have measured SBF distances to 16 galaxies in the vicinity of the LG (Jerjen, Rekola, Takalo, et al. (2001); Rekola, Jerjen \& Flynn (2005)). Dwarf ellipticals DDO 44, KK98 77 and DDO 71 were used to calibrate the SBF method for dEs. Our distances of $27.52 \pm 0.15 \mathrm{mag}$ (or $3.2 \pm 0.2 \mathrm{Mpc}$ ) for DDO 44, $27.71 \pm 0.15 \mathrm{mag}$ (or $3.5 \pm 0.3 \mathrm{Mpc}$ ) for KK98 77 and $27.72 \pm 0.15 \mathrm{mag}$ (or $3.5 \pm 0.3 \mathrm{Mpc}$ ) for DDO 71 agreed with existing 
TRGB distances confirming previously established group memberships of these galaxies (in groups of NGC 2403, M81 and M81 respectively). Similarily KDG 61 and UGC 5442 had existing TRGB distances and BTS 128 an SBF distance; our distances of $27.61 \pm 0.17 \mathrm{mag}$ (or $3.3 \pm 0.3 \mathrm{Mpc}$ ) for KDG $61,27.74 \pm 0.18 \mathrm{mag}$ (or $3.5 \pm 0.3 \mathrm{Mpc}$ ) for UGC 5442 and $31.02 \pm 0.25 \mathrm{mag}$ ( or $16.0 \pm 1.9 \mathrm{Mpc}$ ) for BTS 128 agreed with all of them (placing these galaxies in groups of M81, M81 and Coma I respectively). UGC 7639 had a rough distance based on brightest blue and red stars. We found a slightly smaller distance of $29.27 \pm 0.16 \mathrm{mag}$ (or $7.1 \pm 0.6 \mathrm{Mpc}$ ), with no consequence to its assumed group membership in Canes Venatici II cloud.

The S0 galaxy NGC 4150 had an earlier SBF distance, which we were able to confirm. The distance of $30.79 \pm 0.11 \mathrm{mag}$ ( or $14.4 \pm 0.7 \mathrm{Mpc}$ ) places NGC 4150 to the outskirts of Virgo cluster. Its Local Group barycentric radial velocity of $v_{L G}=207 \mathrm{~km} \mathrm{~s}^{-1}$ is, however, very low for its distance. Solanes, Sanchis, Salvador-Solé, et al. (2002) have found that most Virgo galaxies in the region closest to us, corresponding to our distance to NGC 4150, have high radial velocities outward from the cluster centre. The low radial velocity of NGC 4150 can thus be explained with group dynamics.

We produced completely new distances to the remaining galaxies. The distances are $30.11 \pm 0.18 \mathrm{mag}$ ( or $10.5 \pm 0.9 \mathrm{Mpc}$ ) for UGC 4998 (in the background of M81 group), $27.44 \pm 0.18 \mathrm{mag}$ (or $3.1 \pm 0.3 \mathrm{Mpc}$ ) for DDO 113 (in Canes Venatici I cloud), $29.12 \pm$ $0.18 \mathrm{mag}$ (or $6.7 \pm 0.6 \mathrm{Mpc}$ ) for UGC 7356 (in Canes Venatici I cloud), $28.11 \pm 0.15 \mathrm{mag}$ (or $4.2 \pm 0.3 \mathrm{Mpc}$ ) for UGC 1703 (a companion of NGC 784), 29.01 $\pm 0.27 \mathrm{mag}$ (or $6.3 \pm$ $0.8 \mathrm{Mpc}$ ) for UGCA 200 (possibly a field galaxy), $30.22 \pm 0.17 \mathrm{mag}$ (or $11.1 \pm 0.9 \mathrm{Mpc}$ ) for UGC 5944 (in Leo I group), $30.19 \pm 0.23 \mathrm{mag}$ (or 10.9 $\pm 1.2 \mathrm{Mpc}$ ) for UGC 8799 (in Virgo cluster) with an alternative distance of $30.61 \pm 0.26 \mathrm{mag}$ (or $13.2 \pm 1.7 \mathrm{Mpc}$ ), and $29.60 \pm 0.20 \mathrm{mag}$ (or $8.3 \pm 0.8 \mathrm{Mpc}$ ) for UGC 8882 (in M101 group).

\section{Conclusions}

The SBF distances we have presented continue to support the understanding of the distribution of dwarf galaxies in galaxy groups and intermediate space in the Local Group neighbourhood. They also demonstrate well the feasibility of the Surface Brightness Fluctuation method in determining accurate distances with 2 metre class ground-based telescopes out to the Virgo cluster.

\section{Acknowledgements}

Based on observations made with the Nordic Optical Telescope, operated on the island of La Palma jointly by Denmark, Finland, Iceland, Norway, and Sweden, in the Spanish Observatorio del Roque de los Muchachos of the Instituto de Astrofísica de Canarias.

\section{References}

Baryshev, Yu.V., Chernin, A.D. \& Teerikorpi, P. 2001, A $\& A$ 378, 729

Côté, S., Freeman, K.C., Carignan, C. \& Quinn, P.J. 1997, AJ 114, 1313

Jerjen, H., Binggeli, B. \& Freeman, K.C. 2000, AJ 119, 593

Jerjen, H., Rekola, R., Takalo, L., Coleman, M. \& Valtonen, M. 2001, A\& A 380, 90

Karachentseva, V.E. \& Karachentsev, I.D. 1998, A\&AS 127, 409

Moore, B., Ghigna, S., Governato, F., Lake, G., Quinn, T., Stadel, J. \& Tozzi, P. 1999, ApJL 524,19

Rekola, R., Jerjen, H. \& Flynn, C. 2005, A\& A, 437, 823

Solanes, J. M., Sanchis, T., Salvador-Solé, E., Giovanelli, R. \& Haynes, M. P. 2002, AJ 124, 2440 